\title{
Stress Calculation and Analysis of a 600MW Supercritical Boiler's Steam-water Separator
}

\author{
Haichuan $\mathrm{Xu}^{1, *}$, Haishi Zhang ${ }^{1}$, and Haihua Sun ${ }^{2}$ \\ ${ }^{1}$ POWERCHINA Henan Electric Power Engineering Co., Ltd., Zhengzhou, China \\ ${ }^{2}$ Shananxi Datang New Energy Power Design Co, Ltd., Xi'an, China
}

\begin{abstract}
The steam-water separator of a $600 \mathrm{MW}$ boiler has been chosen as the research object. By theoretical analysis's method and finite element analysis, the stress and secure research has been carried out in the steam-water separator as the thick-walled pressure vessel. For its start-up and normal operation of the working process and features and considering the internal pressure load and thermal stress loading conditions, the initiation of separation stress and security analysis have been carried out about the steamwater separator. The primary stress at the continuum of the cylinder structure can be calculated by the formula, and the calculated results are basically in agreement with the simulated graphic results. And the research work provides a theoretical basis for on-line stress monitoring and life assessment.
\end{abstract}

\section{Introduction}

The supercritical boiler's steam-water separator is the connector between the water wall and the superheater. The normal operation of the steam-water separator is an important guarantee for the power unit's safe operation. And the start-up characteristics of the steam-water separator determine the steam parameters (pressure, temperature) and steam flow. Its stability and flexibility determine the regulation characteristics of once-through boiler under low load. In this paper, the safety of the steam-water separator of $600 \mathrm{MW}$ supercritical unit is analyzed by means of theoretical analysis, which is simplified as a thick-walled cylinder model. And the wall temperature and stress of the steam-water separator are calculated and analyzed under the actual start-up and stop conditions. The safety and strength of the separator are checked under each working condition[1-2].

Each of steam-water separators has six tangential inlet pipes and each a declination of $15^{\circ}$, which are introduced into the top pipe of the steam-water separator. The mixture of steam and water rotates in the separator at a high speed. Steam and water in the vessel are separated by centrifugation and gravity. A dehydrating device is arranged in the middle and upper position of the steamwater separator. The function of the dehydrating device is to eliminate the kinetic energy of the medium rotation and downward, and to make the water level in the starting separator and the water storage tank connected with it stable [3]. The start separator structure views are shown in Figure 1.

The steam-water separator is a three-dimensional cylinder, four separators are arranged above the front part of the boiler. The distance between the separator and the center line of the water wall is $3.575 \mathrm{~m}$, and the distance between two separators is $5.25 \mathrm{~m}$. The outer diameter of the steam-water separator is $610 \mathrm{~mm}$, the wall thickness is $\mathrm{mm}$, and the height of the cylinder is $8.363 \mathrm{~m}$. The separator's material is low alloy steel WB36(Ni-Cu-Mo Steel), which is special for boiler and pressure vessel. Each steam-water separator is suspended on the top plate of the boiler through two suspender[4].

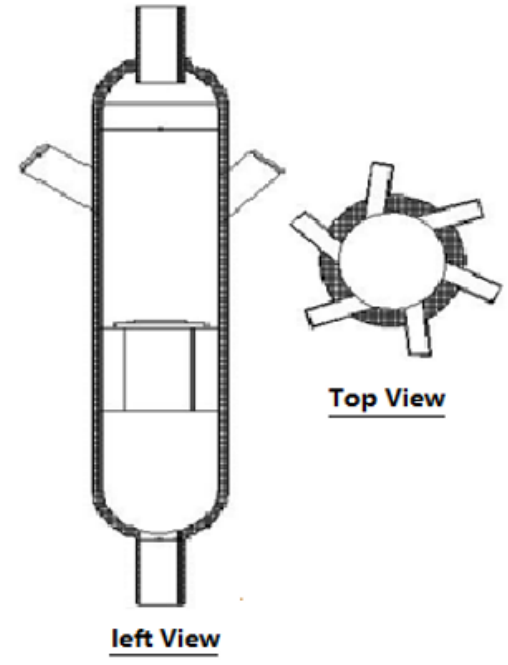

Fig. 1. Model views of the steam-water separator.

\section{Stress Calculation of the Separator}

\subsection{Mechanical Stress Calculation}

From the cold start curve which is shown in Figure 2, six points have been taken to analyze the object. Their respective time, corresponding pressure and temperature are listed in Table 1.

" Corresponding author: whuxhc@163.com 
It can be seen that the area with the greatest mechanical stress occurs on the inner wall or the outer wall, which is based on the the analysis. Then the inner wall and the outer wall are respectively substituted into the formula:

$$
\begin{gathered}
\sigma_{r}=\frac{p_{n} R_{n}^{2}}{R_{w}^{2}-R_{n}^{2}}\left(1-\frac{R_{w}^{2}}{r^{2}}\right) \\
\sigma_{\theta}=\frac{p_{n} R_{n}^{2}}{R_{w}^{2}-R_{n}^{2}}\left(1+\frac{R_{w}^{2}}{r^{2}}\right)
\end{gathered}
$$

It can be concluded that the stresses on the inner and outer walls are expressed as follows:

The inner wall:

$$
\sigma_{r}=-p_{n}, \sigma_{\theta}=p_{n} \frac{k^{2}+1}{k^{2}-1}, \sigma_{m}=0
$$

The outer wall:

$$
\sigma_{r}=0, \sigma_{\theta}=p_{n} \frac{2}{k^{2}-1}, \sigma_{m}=0
$$

In formula(3)\&(4):

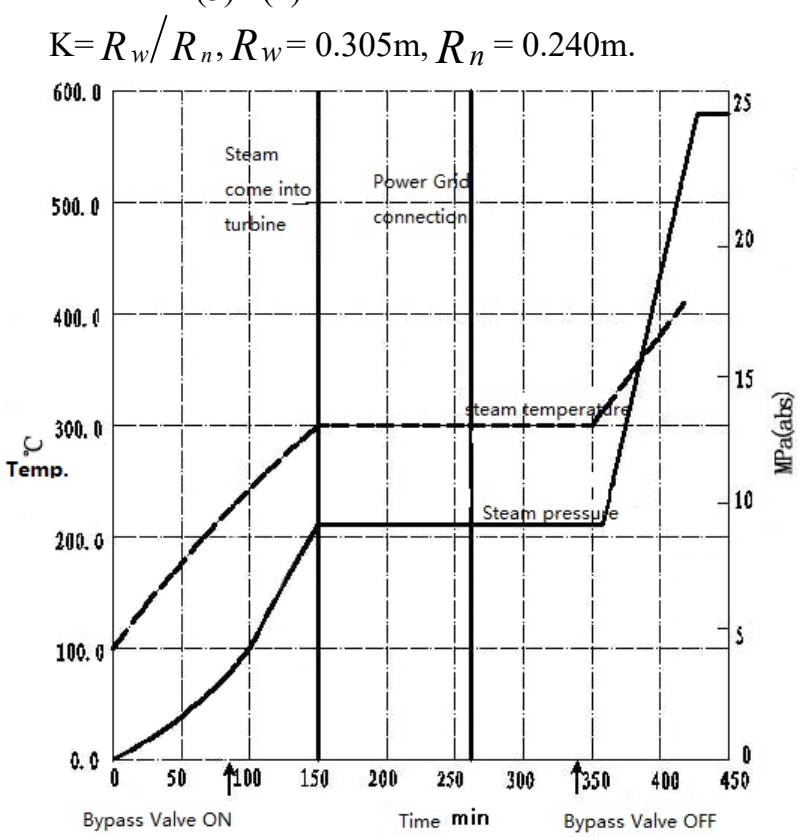

Fig. 2. The cold start-up curve of the power unit.

The parameters in Table 1 are substituted into the formula (3) \& (4).

Table 1. Parameters related to the analysis state.

\begin{tabular}{|c|c|c|c|}
\hline \multirow{2}{*}{ State } & \multicolumn{3}{|c|}{ Operating Conditions } \\
\cline { 2 - 4 } & Time/r/m & Pressure(MP & Temnerature \\
\hline 1 & 70 & 1667 & 203056 \\
\hline 2 & 110 & 5278 & 267383 \\
\hline 3 & 150 & 8920 & 302744 \\
\hline 4 & 350 & 8920 & 342744 \\
\hline 5 & 414 & 22064 & 41399 \\
\hline 6 & 423 & 24167 & 41399 \\
\hline
\end{tabular}

Then the mechanical stress at each state point of the steam-water separator can be calculated, which is shown as Table 2 .
Table 2. Mechanical stress at each state point of the separator.

\begin{tabular}{|l|l|c|c|l|}
\hline \multicolumn{2}{|l|}{ State } & $\sigma_{\theta}(\mathbf{M p a})$ & $\sigma_{r}(\mathbf{M p a})$ & \multirow{2}{*}{$\sigma_{m}$ (Mpa) } \\
\hline \multirow{2}{*}{1} & Inner wall & 7.088 & -1.667 & \multirow{2}{*}{0} \\
\cline { 2 - 4 } & Outer wall & 5.421 & 0 & \\
\hline \multirow{2}{*}{2} & Inner wall & 22.442 & -5.278 & \multirow{2}{*}{0} \\
\cline { 2 - 4 } & Outer wall & 17.164 & 0 & \\
\hline \multirow{2}{*}{$3 /$} & Inner wall & 38.819 & -8.92 & \multirow{2}{*}{0} \\
\cline { 2 - 4 } 4 & Outer wall & 29.007 & 0 & \multirow{2}{*}{0} \\
\hline \multirow{2}{*}{5} & Inner wall & 93.815 & -22.064 & \multirow{2}{*}{0} \\
\cline { 2 - 5 } & Outer wall & 71.751 & 0 & \\
\hline \multirow{2}{*}{6} & Inner wall & 102.757 & -24.167 & \multirow{2}{*}{0} \\
\cline { 2 - 5 } & Outer wall & 78.590 & 0 & \\
\hline
\end{tabular}

\subsection{Thermal Stress Calculation}

The analysis shows that the maximum point of thermal stress also occurs at the inner wall or the outer wall. The thermal stress of the inner and outer walls is expressed as follows [5]:

The inner wall $\left(r=R_{n}\right)$ :

$$
\left.\begin{array}{l}
{ }_{T} \sigma_{r}=0, \\
{ }_{r} \sigma_{\theta}={ }_{r} \sigma_{z}=\frac{\alpha E}{1-\mu}\left[\frac{2}{R_{w}^{2}-R_{n}^{2}} \int_{R_{n}}^{R_{w}} T(r) r d r-T\left(R_{n}\right)\right]
\end{array}\right\}
$$

The outer wall $\left(r=R_{w}\right)$ :

$$
\left.\begin{array}{l}
{ }_{T} \sigma_{r}=0, \\
{ }_{T} \sigma_{\theta}={ }_{r} \sigma_{z}=\frac{\alpha E}{1-\mu}\left[\frac{2}{R_{w}^{2}-R_{n}^{2}} \int_{R_{n}}^{R_{w}} T(r) r d r-T\left(R_{w}\right)\right]
\end{array}\right\}
$$

Equation of temperature field:

$$
t(r, \tau)=t_{0}+V_{\tau}+\frac{V}{4 a}\left(r_{2}-R_{1}^{2}-2 R_{2}^{2} \ln \frac{r}{R_{1}}\right)
$$

The resultant as follows:

The inner wall:

$$
\left.\begin{array}{l}
{ }_{T} \sigma_{r}=0, \\
{ }_{T} \sigma_{\theta}={ }_{T} \sigma_{z}=-0.0015894 \frac{\alpha E}{1-\mu} \frac{V}{a}
\end{array}\right\}
$$

The outer wall:

$$
\left.\begin{array}{l}
{ }_{T} \sigma_{r}=0, \\
{ }_{T} \sigma_{\theta}={ }_{T} \sigma_{z}=0.00070216 \frac{\alpha E}{1-\mu} \frac{V}{a}
\end{array}\right\}
$$

In the formula (8) and (9): $\alpha$ is linear expansion coefficient; $\mu$ is Poisson's ratio, equal to 0.3 ; $\mathrm{E}$ is elastic modulus; $\mathrm{V}$ is temperature change rate; $\mathrm{a}$ is thermal conductivity and $\mathrm{a}=\lambda / \rho \mathrm{c}$.

The radial thermal stress is 0 , and the circumferential stress and axial stress of the inner and outer wall of each state (both are equal) are listed in Table 3.

The mechanical stress and thermal stress after stress concentration are considered.

The maximum stress of the supercritical boiler's steam-water separator is concentrated at the joint of six outgoing pipes, and the inclined cutting is $52^{\circ}$ and the droop is $15^{\circ}$.

The formula as follows: 


$$
k=k_{t}\left[1+(\tan \varphi)^{4 / 3}\right]=1.53 k
$$

So the stress concentration factor of mechanical stress is obtained which is as shown in Table 4.

Table 3. Thermal stress at each state point of the separator.

\begin{tabular}{|c|c|c|c|c|c|}
\hline & State 1 & State 2 & State 3 & State 4 & State 5/6 \\
\hline $\begin{array}{c}\text { Innerwall } \\
(\mathrm{MPa})\end{array}$ & -11.27 & -13.49 & -13.66 & -17.92 & -18.50 \\
\hline $\begin{array}{c}\text { Outwall } \\
(\mathrm{MPa})\end{array}$ & 9.96 & 5.96 & 6.03 & 7.92 & 8.18 \\
\hline
\end{tabular}

Table 4. Stress concentration factor of the separator

\begin{tabular}{|c|c|c|c|c|}
\hline \multirow{2}{*}{} & \multicolumn{2}{|c|}{ Longitudinal section } & \multicolumn{2}{c|}{ Cross section } \\
\cline { 2 - 5 } & Inner Wall & Outer wall & Inner Wall & Outer wall \\
\hline$\sigma_{\theta}$ & 4.743 & 1.836 & 1.53 & 3.213 \\
\hline$\sigma_{r}$ & -0.223 & 0 & -0.223 & 0 \\
\hline$\sigma_{z}$ & 0.306 & 1.53 & -0.306 & 3.978 \\
\hline
\end{tabular}

\subsection{Stress safety analysis}

As the mechanical stress and thermal stress of thick wall vessel are secondary stress, they can be directly added to obtain the comprehensive stress value. And in order to facilitate the stress safety calculation, the stress intensity $\mathrm{S}_{3}$ and the total stress $\mathrm{S}_{4}$ are calculated as shown in Table 5 .

According to Table 4 . and the material yield limit change rule, the most dangerous point is in the inner wall of the longitudinal section of State 6. The WB36's yield strengthos is $441 \mathrm{MPa}$.

According to the formula $[\sigma]=\sigma_{\mathrm{s}} / \mathrm{n}_{\mathrm{s}}$, the allowable stress $\sigma$ is $294 \mathrm{MPa}[6]$.

The $\mathrm{S}_{3}$ is $487.375 \mathrm{Mpa}$ and the $\mathrm{S}_{4}$ is $475.879 \mathrm{Mpa}$ for the $\sigma_{\max }$ section here, and these values are greater than allowable stress. It means that the third strength theory and the fourth strength theory are not satisfied. The stability refers to the continuous cycle of plastic deformation in the process of repeated changes in load, temperature, etc. According to the stability test:

$$
\left(S_{3}=487.375 \mathrm{Mp}\right) \leq(3[\sigma]=661.5 \mathrm{Mp})
$$

\section{Calculation results and analysis of primary stress of the separator}

In order to analyze the influence of the working fluid pressure's change on the primary stress of the steamwater separator, the ANSYS software is used to analyze and simulate the primary stress of the steam-water separator. And the process of calculation is similar to the thermal stress. When the software is used to calculate the primary stress, the corresponding displacement constraints must be loaded on the analysis model.

The displacement constraints on the upper and lower sections. And the model in the direction is the Y-axis, the $\mathrm{X}$-axis is on the left and right sections of the model, and symmetric displacement constraint is in the $\mathrm{Z}$ direction. The pressure acting on the inner wall is equivalent to the saturated pressure of the working medium. The uniform action of the working medium's saturated pressure on the inner wall of the starting separator cylinder. And the steam-water leading-in tube will produce an axial tension force[7-8].

The maximum distribution of the steam-water separator's primary stress is on the upper part of the internal phase connection of the cut-in part. The stress at the junction of the outer wall is also greater than that at other parts. The stress distribution gradient at the inlet of the steam-water separator is larger, and there is a prominent stress concentration at this part.

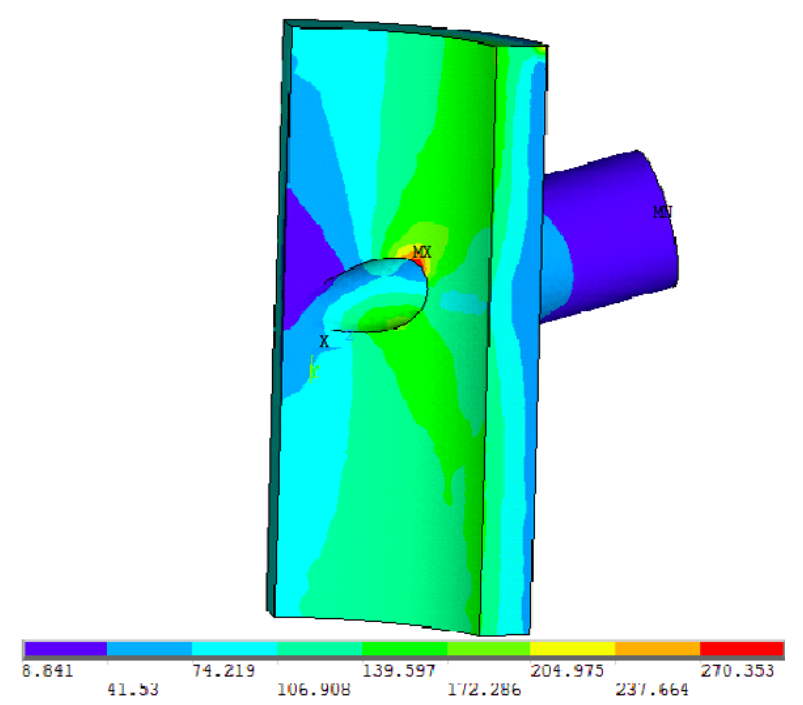

Fig. 3. Distribution diagram I of equivalent stress.

Fig. 3 and Fig. 4 both show the variation of stress with time at the point of maximum stress at the start of the separator during cold start-up. It can be seen that the change trend of the primary stress of the steam-water separator is basically the same as the pressure of the working medium. And the stress is the minimum at the beginning of the cold start-up at the end of the start-up process.

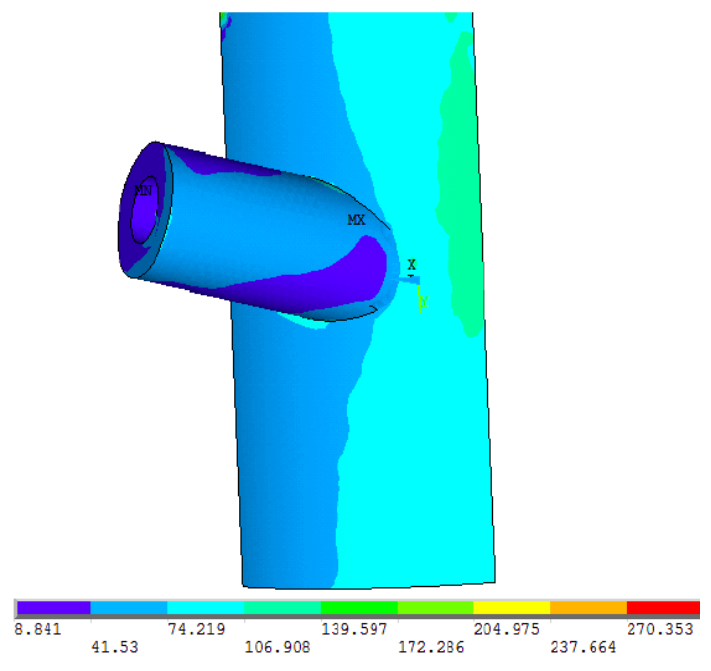

Fig. 4. Distribution diagram II of equivalent stress. 


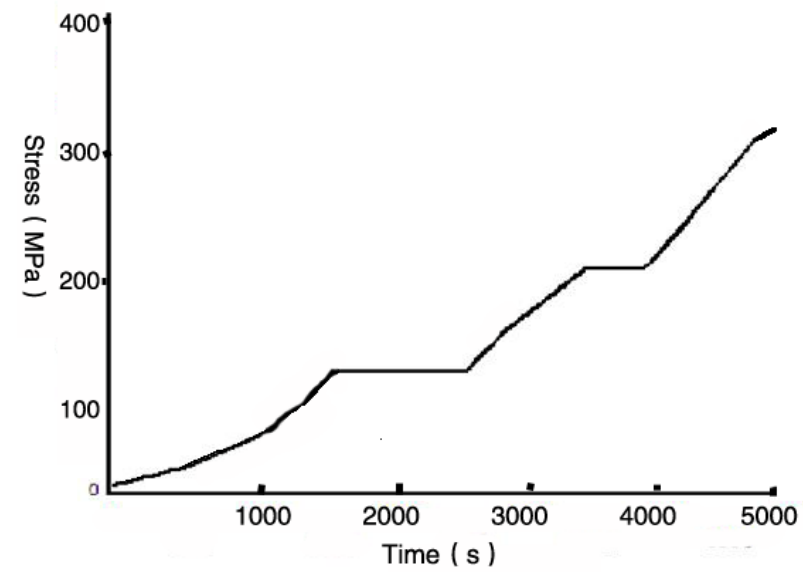

Fig.5. Variation curve of maximum point stress of primary stress with time

According to the post-processing analysis, the trend of the primary stress and the maximum stress point in the other parts of the steam-water separator are basically the same. The primary stress of the steam-water separator is mainly affected by the pressure of the working medium, and the maximum primary stress is mainly concentrated on the connecting part of the steam-water separator cylinder. The magnitude of peak stress is influenced by both thermal stress and primary stress[9].

\section{Conclusion}

The analysis of the supercritical boiler's steam-water separator shows that the research object is safe. Although it is limited by the stress intensity theory, it is also a reasonable application of the stress classification theory and makes full use of the potential of materials. According to the data analysis, the main influence stress of the research object is mechanical stress, especially near the maximum stress (state 6).

However, when the pressure is very small, thermal stress can also play a major role (State 1). When the start-up generator state is abnormal or thermal shock occurs, which makes the temperature change rate very high, the value of thermal stress must not be underestimated. These calculation and analysis work provide effective theoretical calculation support for fault diagnosis and life analysis system of Steam-water separator, and it have certain guiding significance for optimal design and type selection of steam-water separator.

The primary stress of start-up separator is caused by the internal pressure of working medium, and the magnitude of primary stress increases with the increase of working medium pressure. The primary stress at the continuum of the cylinder structure can be calculated by the formula, and the calculated results are basically in agreement with the simulated graphic results.

\section{References}

1. Zhen. Y, SHI Y. Q. Structural strength and life expectancy of the $1913 \mathrm{t} / \mathrm{h}$ supercritical boiler's steam-water separator. Journal of Power Engineering. 26, 4 (2006)

2. Song Z. C, He Z. H, Duan J. X, et al. Investment analysis and forecast report of China power equipment industry from2014-2018, Shenzhen: The consultant. 20-50(2018).

3. Zhu B. P. The fabrication and inspection of the ultra-supercritical separator. Boiler Technology . 40, 63- 66 (2006)

4. Jiang Y. C, Deng T. T, Zhang. Y, et al. Analysis on economic operation mode of $600 \mathrm{MW}$ fossil-fired generating during peak shaving low load operation. Turbine Technology. 1, 61-64(2015)

5. Zhu G. M, Jiao Q. F, LI. M, et al. Research on economic efficiency of low load operation in large thermal power units, Hunan Electric Power. 28, 2426(2008)

6. Qian S. W. Heat exchanger design handbook, M.Beijing, Chemical Industry Press. 55-99(2002)

7. Du Y. Y, Feng W. Z. Research of properties of frequency regulation based on the flexible extraction technology, East China Electric Power, 42, 19441949(2014)

8. Yan J. J, Huang J. T. Zhang. K, et al. Power plant thermal systems and equipment, Xi'an: Xi'an Jiaotong University Press, 313-321 (2003)

9. Li. N,Yan W. P,Ye. F, et al. Creep-fatigue interaction life prediction for P91 steel based on improved Elman neural network model, Advanced Materials Research, 674- 677 (2017) 